\title{
Synthesis of Narrowly Dispersed Block Copolymer of Butyl Acrylate and Acrylic Acid in a Solution Acquiring Colloidal Properties during Cooling
}

\author{
N. Yu. Shushunova ${ }^{a, *}$, B. B. Troitskii ${ }^{a}$, S. A. Chesnokov ${ }^{a}$, T. A. Kovylina ${ }^{a}$, and A. N. Konev ${ }^{a}$ \\ ${ }^{a}$ Razuvaev Institute of Organometallic Chemistry, Russian Academy of Sciences, Nizhny Novgorod, 603950 Russia \\ *e-mail: shushunova@iomc.ras.ru
}

Received March 31, 2020; revised July 31, 2020; accepted September 8, 2020

\begin{abstract}
The homophase polymerization of acrylic acid is carried out in a solution of poly(butyl acrylate) trithiocarbonate (PBATC) in a mixed solvent 2-propanol-water. The phase diagram of the three-component PBATC-2-propanol-water system at $22^{\circ} \mathrm{C}$ is obtained. The homophase region of the compositions of semidilute solutions is determined. The homophase polymerization of AA in the three-component system at $70^{\circ} \mathrm{C}$, in which the mixed solvent with respect to PBATC is similar in thermodynamic quality to the $\theta$-solvent at $22^{\circ}$, yields solutions of the narrowly dispersed block copolymer butyl acrylate-acrylic acid. The content of the block copolymer in solutions is $15-23 \%$, the MW of the copolymer is $10-20 \times 10^{3}$, and the polydispersity coefficient is 1.12-1.16. As evidenced by dynamic light scattering, under normal conditions, the solutions contain nanoparticles with a diameter of up to $50 \mathrm{~nm}$ with a narrow size distribution.
\end{abstract}

DOI: $10.1134 / \mathrm{S} 156009042101005 \mathrm{X}$

\section{INTRODUCTION}

Synthetic amphiphilic block copolymers with surface activity are widely used as components of a wide variety of chemical products from motor oils and drilling fluids in oil production to household cleaning products and cosmetics [1]. Associates of amphiphilic block copolymers are of great practical interest. Owing to sensitivity to changes in temperature and $\mathrm{pH}$ of solution they are used in various fields of medicine and biotechnology as nanocarriers of biologically active substances and drugs for their delivery to the human body [2]. Also known is the use of such polymer nanoparticles as templates for obtaining hollow silicon dioxide particles in creation of antireflection coatings on silicate glass [3]. In this case, an increased requirement is imposed on the size of nanoparticles (no more than $100 \mathrm{~nm}$ ) and their size uniformity. Such particles are formed in a colloidal solution of amphiphilic macromolecules of narrowly dispersed block copolymers in a selective solvent that selectively dissolves one of the blocks. At present, one of the simplest and most affordable methods for preparing such a colloidal solution is heterophase-controlled polymerization occurring by the reversible addition-fragmentation chain transfer (RAFT) mechanism, which makes it possible to simultaneously control the molecular characteristics of the synthesized block copolymer and tune the size and morphology of the resulting micelles in the nanoscale.
However, this heterophase method has a limitation in cases where it is impossible to implement conditions for RAFT polymerization in a given medium. In particular, in dispersion RAFT polymerization it is often difficult to choose a selective solvent in which all reagents are readily soluble but which is selective with respect to the blocks of the synthesized block copolymer. These requirements limit the choice of medium. For example, the aqueous dispersion RAFT polymerization allows one to obtain a colloidal solution only for a small number of water-soluble monomers which form insoluble polymers during polymerization [4]. It is impossible to carry out the dispersion RAFT polymerization of various styrenes in alcohols [5]. Despite the fact that water and alcohols are the most popular media for the heterophase RAFT process, many attempts have recently been made to search for new solvents. These are long-chain $n$-alkanes $[6,7]$, mineral oil [8], ionic liquids [9], and poly(ethylene glycol) [10]. However, either the use of these solvents is not economical or they do not fully meet requirements of the polymerization preparation of the colloidal solution. It should be noted that limitations also apply to the choice of a polymeric RAFT agent, to which the role of a stabilizer of colloidal nanoparticles is uniquely assigned.

The problem can be solved by obtaining a colloidal solution of a block copolymer in a medium in which a homophase solution is formed at elevated temperatures of polymerization. When such a solution is 
cooled, the solvent becomes selective with respect to the blocks of the copolymer, inducing the self-organization of its macromolecules into nanoobjects. As known [11], the compatibility of polymers in solution deteriorates if the solvent is poor for one of the polymers. This leads to a decrease in the delamination limit of a given polymer mixture, that is, to a decrease in the concentration range of existence of single-phase polymer mixtures in solution upon passing to a poor solvent for one of them.

Similar regularities are typical for solutions of block copolymers with the difference that during phase separation in such systems, owing to the presence of covalent bonds between blocks, the spontaneous aggregation of macromolecules can occur to form core-shell microparticles and nanoparticles: the core of the particles is formed by lyophobic blocks of the block copolymer, and the stabilizing shell is formed by the lyophilic block. As a result of phase separation, the homophase solution of the block copolymer becomes the colloidal solution.

Earlier [12], it was assumed that the colloidal solution of the amphiphilic block copolymer can be prepared using the high-temperature synthesis of a lyophilic polymer in a homophase solution of a polymeric RAFT agent, the concentration of which is close to critical under normal conditions. This approach was tested in preparation of the colloidal solution of butyl acrylate (BA) copolymer with acrylic acid (AA) in the mixed solvent 2-propanol (IPA)-water in the presence of poly(butyl acrylate) trithiocarbonate (PBATC). As a consequence, the colloidal solutions of narrowly dispersed BA-AA copolymers with dispersion $D=1.1-1.6$ were obtained.

However, the reaction mechanism remained unclear. This work is aimed at studying the mechanism of the process and identifying conditions (including the parameters of the medium) necessary for the synthesis of the colloidal solution of the amphiphilic block copolymer BA-AA with a given particle size.

\section{EXPERIMENTAL}

Monomers $n$-butyl acrylate (NevaReaktiv) and acrylic acid (Acros Organics) were distilled in vacuum. Solvents were 2-propanol (special purity grade, Ekos) and water (bidistillate). Polymerization initiators AIBN (Reakhim) (purified by double recrystallization from methanol) and reagent grade ammonium persulfate (APS, Reakhim) were used without additional purification).

Dibenzyl trithiocarbonate (DBTC) was synthesized according to a modified procedure [13].

BA-AA diblock copolymers were synthesized by the two-stage RAFT polymerization. At the first stage, narrowly dispersed poly(butyl acrylate) was synthesized in the presence of AIBN and the RAFT agent
DBTC at $60^{\circ} \mathrm{C}$. The polymer yield was 95-98\%. At the second stage, the obtained poly(butyl acrylate) (PBA) containing trithiocarbonyl groups participated as a polymeric RAFT agent in the polymerization of AA, which was carried out in the IPA-water mixture at $70^{\circ} \mathrm{C}$. APS taken in the amount of 0.004$0.016 \mathrm{~mol} / \mathrm{L}$ was used as an initiator. The AA concentration was $0.38-3.40 \mathrm{~mol} / \mathrm{L}$. The PBATC concentration was varied from 0.003 to $0.008 \mathrm{~mol} / \mathrm{L}$ depending on the molecular weight of the polymer. The copolymer yield was $85-95 \%$.

The AA conversion was determined by the gravimetric method. A mixture of monomer AA, PBATC, IPA, water, and APS with the known composition was placed in several ampoules and degassed in vacuum via three freeze-pump-thaw cycles. The ampoules were sealed off and placed in a thermostat at $70^{\circ} \mathrm{C}$. At specified time intervals the ampoules were removed, cooled with liquid nitrogen, and dried in vacuum to a constant weight to remove the residual monomer.

To obtain a mechanical mixture, PAA and PBA homopolymers were synthesized in a model experiment. PAA was synthesized at $60^{\circ} \mathrm{C}$ in IPA ( $\left.75 \mathrm{vol} \%\right)$ containing $0.016 \mathrm{~mol} / \mathrm{L}$ of DBTC and $0.0057 \mathrm{~mol} / \mathrm{L}$ of AIBN. PBA was polymerized out in bulk in the presence of $0.029 \mathrm{~mol} / \mathrm{L}$ of DBTC and $0.0029 \mathrm{~mol} / \mathrm{L}$ of AIBN at $60^{\circ} \mathrm{C}$.

The molecular weight characteristics of the copolymers were analyzed by GPC using a Knauer Smartline chromatograph equipped with Phenogel Phenomenex $5 \mathrm{u}(300 \times 7.8 \mathrm{~mm})$ columns with an average pore diameter of $10^{4}$ and $10^{5} \AA$ and a refractometer as a detector. THF served as a mobile phase, the flow rate was $2 \mathrm{~mL} / \mathrm{min}$, and $T=40^{\circ} \mathrm{C}$. Calibration was performed using polystyrene standards with MW values ranging from $2.7 \times 10^{3}$ to $2.57 \times 10^{6}$. Before measurements, the BA-AA copolymers were methylated with diazomethane, as described in [14].

The size of particles was measured by dynamic light scattering on a Brookhaven NanoBrook Omni particle size analyzer. To improve the measurement accuracy, the optimal concentration of aqueous-alcohol polymer solutions was selected so that the average value of the light intensity was in the range of $100-400 \mathrm{kcps}$. Before loading in cells, the aqueous-alcohol solutions were filtered through Chromafil Xtra CA-20/25 syringe filters with a pore size of $0.2 \mu \mathrm{m}$. A cuvette containing $3 \mathrm{~mL}$ of solution was placed in a cell thermostatted to $25^{\circ} \mathrm{C}$; five measurements of each sample were carried out (the time of one measurement was $300 \mathrm{~s})$. The results were averaged over the mode.

Intrinsic viscosity $[\eta]$ was determined in an Ubbelohde viscometer at 22 and $30^{\circ} \mathrm{C}$ according to [15].

The phase diagram of the three-component system at a temperature of $22^{\circ} \mathrm{C}$ was obtained by the cloud point method [16].

The solutions of DBTC and AIBN in IPA and benzene were analyzed by HPLC on a Knauer liquid chro- 


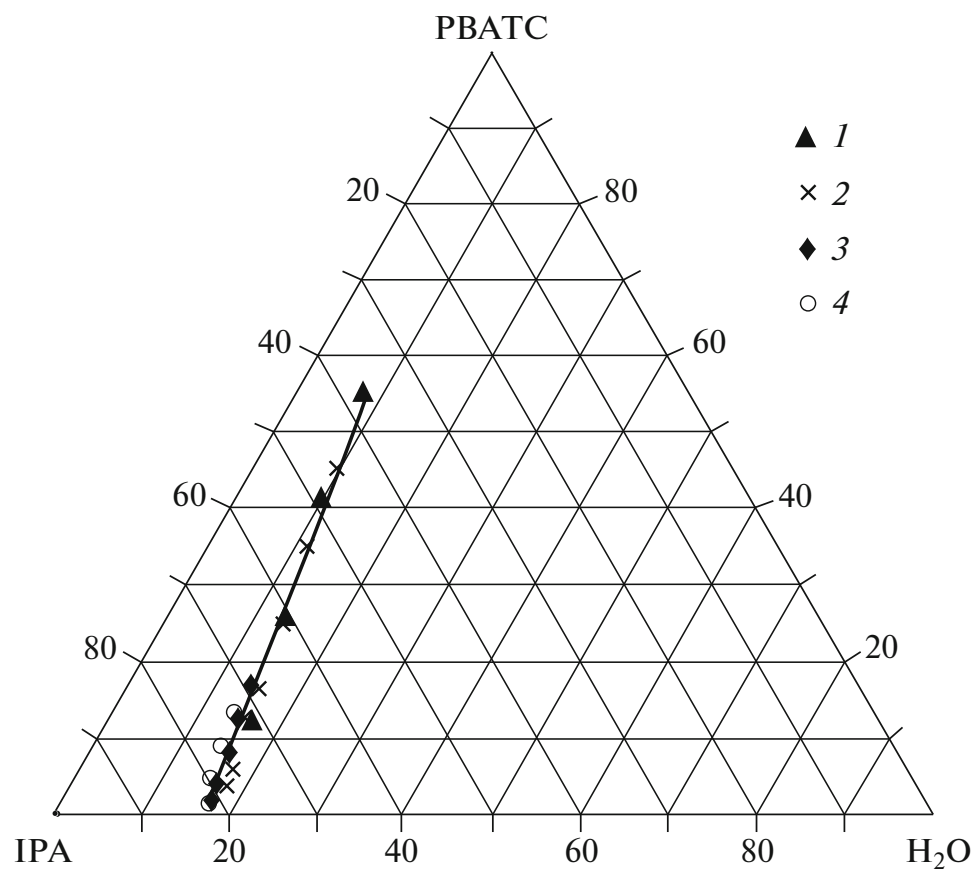

Fig. 1. Phase diagram of the IPA- $\mathrm{H}_{2} \mathrm{O}-\mathrm{PBATC}$ three-component system. The molecular weight of PBATC, $M_{\mathrm{n}} \times 10^{-3}$ : (1) 13.3 , (2) 30 , (3) 54.4 , and (4) $119 . T=22^{\circ} \mathrm{C}$.

matograph (Knauer, Germany) at a temperature of $19 \pm 1{ }^{\circ} \mathrm{C}$ using a UV spectrophotometer $(\lambda=254 \mathrm{~nm})$ as a detector. Reaction mixtures were separated on a stainless steel column $(120 \times 4 \mathrm{~mm})$ filled with a Eurospher-100 C18 sorbent (Knauer, Germany) with a particle size of $5 \mu \mathrm{m}$. The mobile phase was an acetonitrile-water mixture $(8: 2)$, the volume flow rate of the eluent was $1.5 \mathrm{~mL} / \mathrm{min}$, and the volume of the injected sample was $20 \mu \mathrm{L}$. Special purity grade acetonitrile (Kriokhrom, St. Petersburg), bidistilled water, and the eluent were degassed in vacuum before analysis. Chromatograms were processed using the MultiChrom v.1.52i software.

\section{RESULTS AND DISCUSSION}

As already noted, the solutions of block copolymers are characterized by worsening of the compatibility of blocks if the solvent is poor for one of them [11]. In this case, the delamination limit of the block copolymer solution (critical micelle concentration) is lower than the delamination limit of the solution of the poorly soluble block-forming polymer. Using this effect, we tried to create critical conditions for delamination of the BA-AA block copolymer solution at room temperature so that the solution obtained at $70^{\circ} \mathrm{C}$ under homophase conditions was heterophase after synthesis and cooling to room temperature. As was shown in [12], the polymerization of AA mediated by PBATC yields the colloidal solution of the amphiphilic block copolymer BA-AA in the mixed solvent IPA $-\mathrm{H}_{2} \mathrm{O}$ at $22^{\circ} \mathrm{C}$. The mixed solvent IPA- $\mathrm{H}_{2} \mathrm{O}$ dis- solves PAA unlimitedly. At the same time, for PBATC, it is a mixture of a precipitant (water) and a solvent (IPA), the composition of which determines the solubility of PBATC. Since the polymerization of AA occurs in the medium of the three-component system PBATC-IPA- $\mathrm{H}_{2} \mathrm{O}$, the phase diagram of this threecomponent system at $22^{\circ} \mathrm{C}$ was obtained (Fig. 1) to ascertain the homophase region of component ratios.

In the diagram, each model mixture differing in the molecular weight of PBATC is only a part of the boundary curve, since owing a high viscosity of its concentrated solutions, determination of the cloud point is hardly possible. It can be seen that, over the entire studied range of polymer molecular weights from $13.3 \times 10^{3}$ to $119 \times 10^{3}$, all compositions form one boundary curve, to the left of which there is the homophase region. It follows from the nature of the boundary curve that the composition of the mixed IPA-water solvent changes little with an increase in the PBATC concentration.

To assess compositions with the poor solubility of PBATC in the region of semidilute solutions (depending on molecular weight, its content in the three-component system ranged from 5 to $20 \%$ ), the data obtained in the region of dilute solutions were used. The thermodynamic quality of the mixed solvent with respect to PBATC was roughly estimated in a dilute solution containing $14.8 \%$ water, which is $\sim 3 \%$ smaller than the critical value.

Figure 2 shows the dependence of the intrinsic viscosity on the molecular weight of the polymer in 


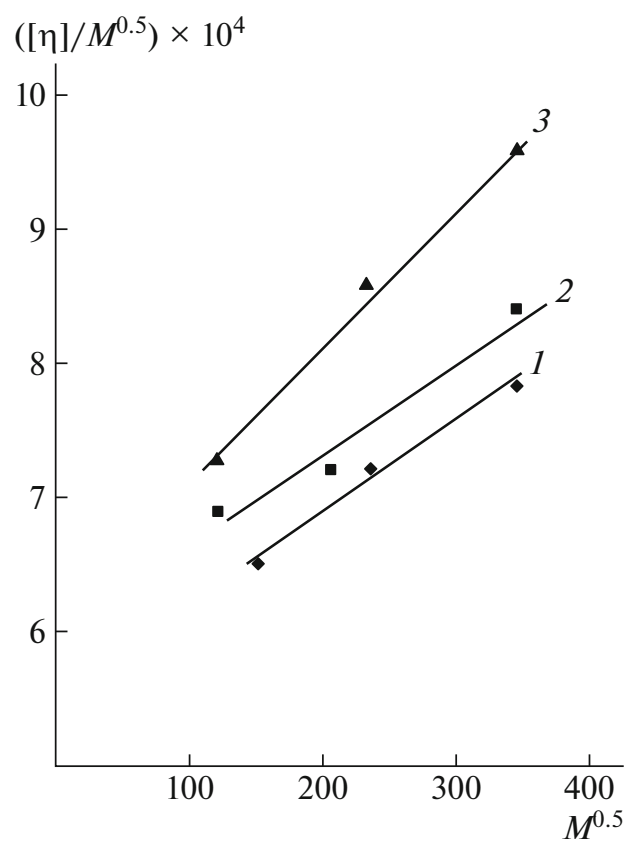

Fig. 2. The intrinsic viscosity of PBATC solutions in the mixed solvent IPA $-\mathrm{H}_{2} \mathrm{O}$ as a function of the molecular weight of PBATC in Stockmayer-Fixman coordinates at a water content of (1) 14.8 and $(2,3) 17.8 \mathrm{wt} \%((3)$ with the addition of $1 \mathrm{wt} \% \mathrm{AA}) . T=(1) 22$ and $(2,3) 30^{\circ} \mathrm{C}$.

Stockmayer-Fixman coordinates [17] which was obtained in the mixed solvent IPA- $\mathrm{H}_{2} \mathrm{O}$ at a temperature of $22^{\circ} \mathrm{C}$ (straight line 1). The Huggins parameter of the mixed solvent calculated using the StockmayerFixman equation is $\chi=0.494$. As can be seen, in terms of thermodynamic quality, the mixed solvent of this composition approaches the $\theta$-solvent. This means that the solubility of PBATC in a solvent with the content of water (precipitant) $2-4 \%$ smaller than the critical one is poor and worse than that of PAA.

With an increase in temperature from 22 to $28.5^{\circ} \mathrm{C}$, initially heterophase boundary mixtures become transparent (Table 1). As follows from Fig. 2 (straight line 2), the IPA- $\mathrm{H}_{2} \mathrm{O}$ mixture, which is a precipitant for PBATC at the boundary composition at $22^{\circ} \mathrm{C}$, becomes a solvent at $30^{\circ} \mathrm{C}$. The calculated Huggins parameter is also 0.494. Adding even small amounts of AA (1 wt \%) to the solution improves the dissolving ability of the IPA- $\mathrm{H}_{2} \mathrm{O}$ mixture. The Huggins parameter $\chi$ calculated from the data in Fig. 2 (straight line 3 ) is 0.49 . From this, it follows that the PBATCIPA- $\mathrm{H}_{2} \mathrm{O}$ system has a UCST. At high temperatures, the polymerization of AA in the IPA- $\mathrm{H}_{2} \mathrm{O}-\mathrm{PBATC}$ medium should proceed under homophase conditions.

Using the results obtained for dilute solutions, the composition of the medium for the polymerization of acrylic acid in the region of semidilute solutions (the PBATC content was from 5 to $20 \mathrm{wt} \%$ ) was chosen so that the concentration of water in them was $2-4 \%$ lower than that in the corresponding boundary compositions with the same molecular weight and concentration of PBATC.

Figure 3 shows the kinetic curves of AA polymerization in the PBATC-IPA- $\mathrm{H}_{2} \mathrm{O}$ medium. It is seen that the polymerization of AA proceeds to deep conversions (curves 1,2). Figure 4 presents the GPC curves of polymerization products at different conversions of AA; curve 1 corresponds to the initial PBATC. The polymerization of AA leads to the appearance of a new narrowly dispersed polymer, which corresponds to the main mode on GPC curves $2-7$. This mode has a high molecular weight shoulder due to the initial PBATC which decreases with the conversion of AA and disappears at $100 \%$ conversion (curve 7). It should be noted that, at a degree of AA conversion of above $60 \%$, in addition to the main one, a low molecular weight polymer mode appears, the relative content of which at the final stages of AA polymerization does not exceed $2.6 \%$.

The observed consumption of PBATC during the polymerization of AA makes it possible to assign the main mode to the copolymerization product. However, it is also necessary to consider the option that the resulting PAA can correspond to the main mode. In this case, in Fig. 4, there would a superposition of modes corresponding to the components of the PAAPBA mixture at different conversions of AA. Then, at the final stages of polymerization, the main mode of PAA would increase so much that against its background the mode related to PBA would become invisible (despite the fact that the weight ratio of PAA : PBATC at $100 \%$ AA conversion should be $1: 4$ ).

To test this assumption, PAA with a molecular weight of $M_{\mathrm{n}}=12.7 \times 10^{3}(D=1.45)$ and PBATC with a molecular weight $M_{\mathrm{n}}=31 \times 10^{3}(D=1.1)$ were synthesized. Figure 5 shows the GPC curves of homopolymers PBATC and PAA and their mechani-

Table 1. Temperature dependence of the phase state of the PBATC-IPA- $\mathrm{H}_{2} \mathrm{O}$ system

\begin{tabular}{c|c|c|c|c}
\hline $\begin{array}{c}\text { Boundary composition of } \\
\text { the three-component system } \\
\text { PBATC-IPA- } \mathrm{H}_{2} \mathrm{O}, \mathrm{wt} \%\end{array}$ & $\begin{array}{c}\text { Molecular weight } \\
\text { of PBATC } \\
M_{\mathrm{n}} \times 10^{-3}\end{array}$ & $22^{\circ} \mathrm{C}$ & $28.5^{\circ} \mathrm{C}$ & $30^{\circ} \mathrm{C}$ \\
\cline { 2 - 5 } & 30 & Turbid & Transparent & Transparent \\
\hline $4.1: 78.01: 17.8$ & 119 & Turbid & Transparent & - \\
\hline
\end{tabular}


cal mixture at a weight ratio of $1: 4$ (curves $1,2,3$, respectively). The molecular weights of PAA and PBATC and their weight ratio in the mixture simulate the product of polymerization of AA with PBATC at $100 \%$ AA conversion. (The mixture was methylated before testing, Experimental.) A comparison of curve 7 in Fig. 4 and curve 3 in Fig. 5 shows that the nature of these curves is different. The GPC curve of the mechanical mixture (Fig. 5, curve 3) has a bimodal MWD (the low molecular weight mode corresponds to PAA, and the high molecular weight mode corresponds to PBATC), while the GPC curve of the product of the PBATC-mediated polymerization of AA is unimodal.

The presented data indicate that the main peak in curve 7 (Fig. 4) refers to an individual polymer, which apparently is the BA-AA copolymer.

The microstructure of the obtained polymer product was estimated using the approach proposed in [18]. As is known, as a result of RAFT polymerization mediated by symmetric trithiocarbonates, to which PBATC belongs, the main reaction product is a polymer containing either one or two polymeric substituents at the trithiocarbonate group. To determine the number of substituents, the polymer without the monomer is heated in benzene in the presence of a 100 -fold excess of AIBN at $80^{\circ} \mathrm{C}$ for $2.0-2.5 \mathrm{~h}$. Under these conditions, the polymer radical released upon detachment from the trithiocarbonate group enters into the termination reaction with the cyanoisopropyl radical. If during the synthesis a polymer containing one polymer substituent is produced, then after heating its molecular weight remains almost unchanged. If a polymer containing two polymeric substituents is formed, its molecular weight is halved.

Figure 6a shows the chromatograms of the initial PBATC (curve 1) before and (curve 2) after heating in benzene under the above conditions. It is seen that the polymer has a unimodal distribution before heating. After heating, products with a bimodal MWD are formed. The molecular weight of the low molecular weight fraction is approximately two times less than the molecular weight of the main peak corresponding to the initial polymer before heating. This is evidence that the PBATC molecule contains two polymeric substituents at the trithiocarbonate group close in molecular weight. As shown in [18], polymer radicals arising upon heating as a result of detachment from the thiocarbonate group also enters into the cross-termination reaction with an intermediate, which leads to the appearance of the high molecular weight mode with a higher molecular weight than that of the initial PBATC.

Figure $6 \mathrm{~b}$ shows the chromatograms of the product of AA polymerization mediated by PBATC described above before and after heating. The molecular weight of the main peak does not change, which, as noted above, is typical of the polymer containing one poly-

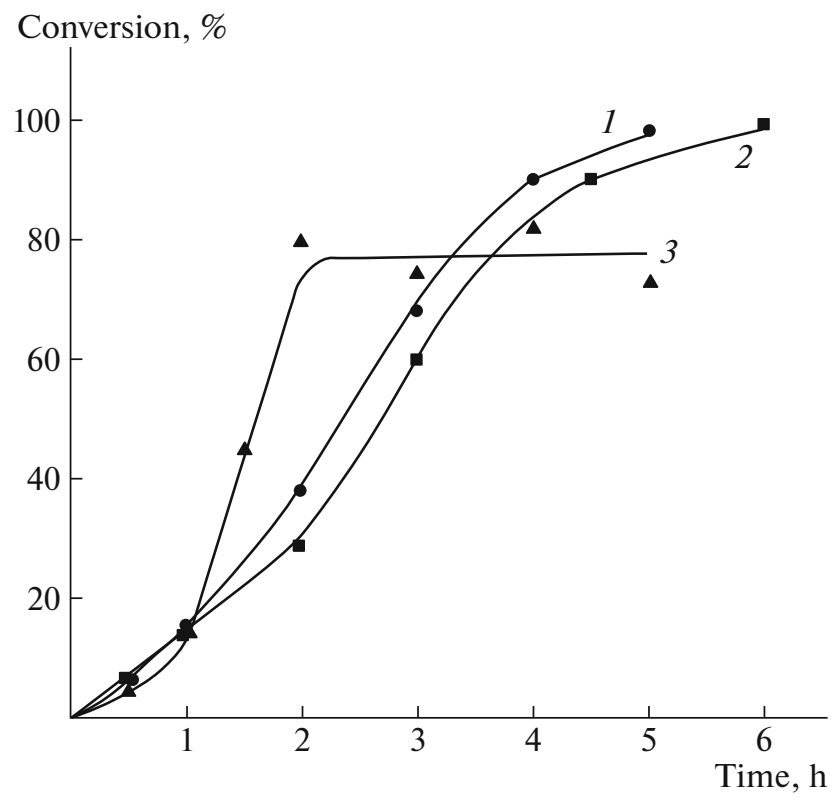

Fig. 3. Kinetic curves of AA polymerization at $70^{\circ} \mathrm{C}$ in the presence of $(1,2)$ PBATC and (3) PBA. Component concentration, mol/L: (1) $[\mathrm{AA}]=0.54,[\mathrm{PBATC}]=0.0042$ $\left(M_{\mathrm{n}}=42.8 \times 10^{3}\right),\left[\mathrm{H}_{2} \mathrm{O}\right]=6.84,[\mathrm{IPA}]=11$, and $[\mathrm{APS}]=$ $0.0044 ;(2)[\mathrm{AA}]=0.36,[\mathrm{PBATC}]=0.0036\left(M_{\mathrm{n}}=30.3 \times\right.$ $\left.10^{3}, M_{\mathrm{w}}=35.7 \times 10^{3}\right),\left[\mathrm{H}_{2} \mathrm{O}\right]=7.26,[\mathrm{IPA}]=11.07$, and $[\mathrm{APS}]=0.0024$; and $(3)[\mathrm{PBA}]=11.9 \mathrm{wt} \%\left(M_{\mathrm{n}}=10.5 \times\right.$ $\left.10^{3}, M_{\mathrm{w}}=37.8 \times 10^{3}\right),[\mathrm{AA}]=0.36,\left[\mathrm{H}_{2} \mathrm{O}\right]=7.26,[\mathrm{IPA}]=$ 11.07 , and $[\mathrm{APS}]=0.0024$.

meric substituent at the thio group. A bimodal molecular weight distribution is observed, the main mode of which belongs to the initial polymer, and the high molecular weight shoulder belongs to the products of the cross-termination of macroradicals and intermediates [18]. The main peak cannot be attributed to either PBATC or the mechanical mixture of BA and AA homopolymers. If we assume that the polymerization of AA mediated by PBATC yields the copolymer PBA-AA with the thio group arranged in the middle of the chain, then after heating the copolymer its molecular weight would decrease by a factor of 2 , since the PAA block would be incorporated into the middle of the chain. This effect is possible only if the resulting copolymer does not contain the thio group.

The above findings are contradictory. On one hand, the main feature of controlled polymerization, namely, an increase in the molecular weight of the resulting polymer with conversion, is absent. On the other hand, the polymer product with dispersion $D$ close to unity can be obtained only under controlled synthesis conditions, which implies the participation of PBATC in the polymerization of AA as a RAFT agent. But this is ruled out because of the absence of the thio group in the resulting polymer. 


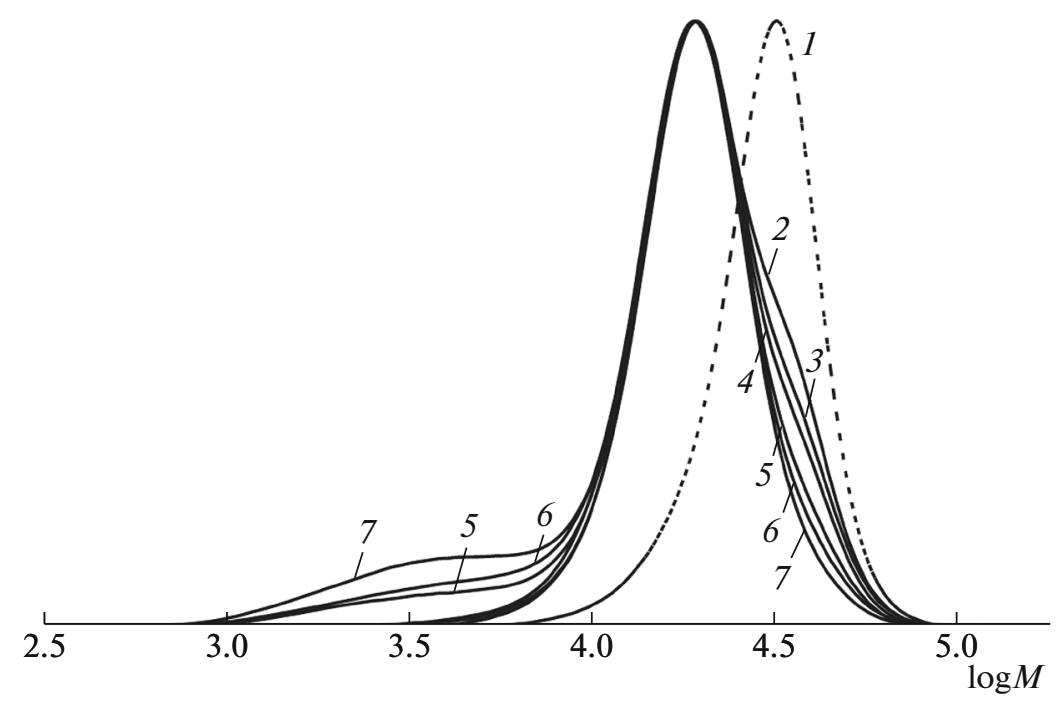

Fig. 4. GPC curves of the product of PBATC-mediated AA polymerization isolated at an AA conversion of (1) 0, (2) 6.9, (3) 14.2 , (4) 26.5, (5) 60, (6) 92, and (7) $100 \mathrm{wt} \%$. The initial concentration of components, mol/L: [AA] $=0.36$, [PBATC] $=0.0036$, $\left[\mathrm{H}_{2} \mathrm{O}\right]=7.26,[\mathrm{IPA}]=11.07$, and $[$ APS $]=0.0024 . T=70^{\circ} \mathrm{C}$.

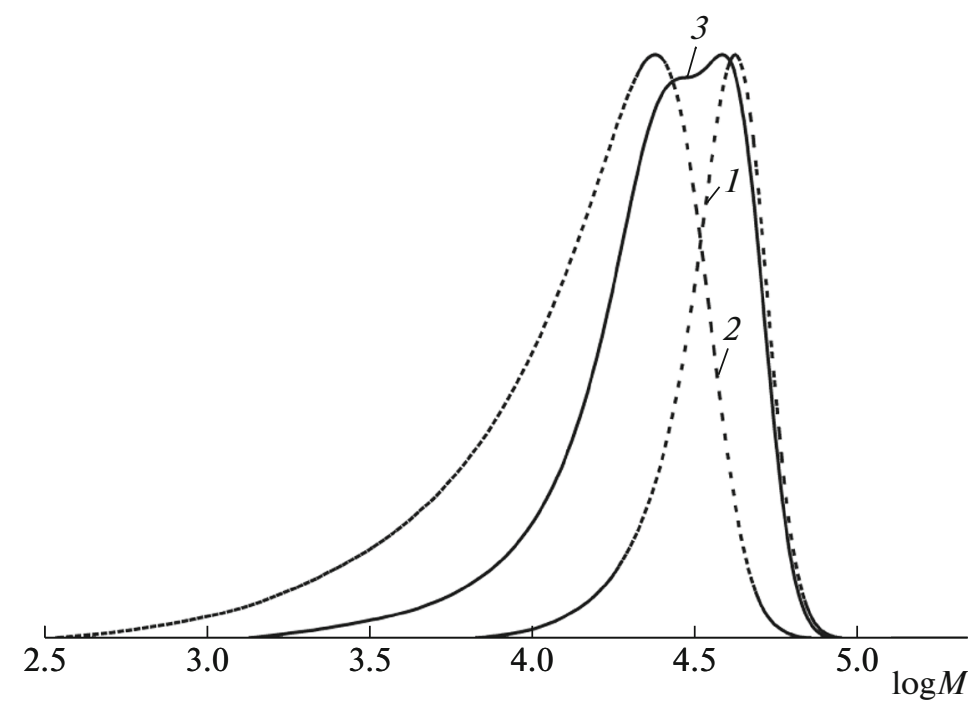

Fig. 5. GPC curves of (1) PBATC and (2) PAA homopolymers and (3) their mechanical mixture at the weight ratio of PAA : $\operatorname{PBATTK}=1: 4$.

The polymerization of AA upon the addition of PBATC proceeds with autoacceleration, which occurs at deep conversions and is caused by the diffusion control of polymerization owing to the high viscosity of the reaction medium. Figure 3 shows the kinetic curves of AA polymerization with the addition of PBATC (curve 2) and PBA obtained by uncontrolled radical polymerization (curve 3 ) with close $M_{\mathrm{w}}$ values $\left(35.7 \times 10^{3}\right.$ and $37.8 \times 10^{3}$, respectively). It can be seen that the gel effect is also observed in the presence of PBA. However, the intensity of autoacceleration is much higher and the degree of completion of AA polymerization is much lower (75-80\%) compared to the polymerization of AA mediated by PBATC. It is known [19] that, during the uncontrolled polymerization of AA in aqueous and alcohol solutions at low initial monomer concentrations, intramolecular chain transfer to the polymer takes place owing to participation of the active secondary radical of the terminal PAA unit in the abstraction of the main-chain hydrogen atom. This leads to the branching and crosslinking of chains and, as a consequence, to a low limiting conversion $[19,20]$. It appears that, in the presence of 
PBATC, this process does not occur, as evidenced by the high polymer yield (98\%).

Figure 7 shows the GPC curves of poly(butyl acrylate) obtained by uncontrolled radical polymerization and products of AA polymerization in its presence isolated 2 and $3 \mathrm{~h}$ after the beginning of the process, the kinetic curve of which is shown in Fig. 4 (curve 3). Curve 1 in Fig. 7 corresponds to the initial PBA; after AA polymerization, the main mode of $\mathrm{PBA}$ acquires a low molecular weight shoulder with a molecular weight of $\sim 10^{3}$ (curves 2,3 ), which are uniquely related to PAA.

The molecular weight of PAA is 3-4 times lower than that of the product of copolymerization of AA with PBATC, as indicated by Fig. 4 and Table 2. This allows one to conclude that the polymerization of AA mediated by PBATC yields the BA-AA copolymer rather than PAA. Since the PBA radical arising during the fragmentation of the PBATC intermediate can become a component of the copolymer only as a finished identical block (Fig. 6), the BA-AA copolymer must be a block copolymer.

As is known [18], RAFT polymerization mediated by symmetric polymeric RAFT agents, such as PBATC, affords triblock copolymers, for example, $\mathrm{P}(\mathrm{BA}-\mathrm{AA})-\mathrm{S}-\mathrm{C}(=\mathrm{S}) \mathrm{S}-\mathrm{P}(\mathrm{AA}-\mathrm{BA})$. In this case, the molecular weight of the copolymer should be higher than that of the initial polymeric RAFT agent. However, as is seen from Table 2, the molecular weight of the block copolymer formed by the PBATC-mediated polymerization of AA is lower than that of the initial RAFT agent. A similar effect was described in the study of 11 copolymers of AA with PBATC of various molecular weights synthesized by the same method [12]. The data show that the pattern found has a general character. The only possible structure with a molecular weight lower than that of the initial PBATC can be only an asymmetric BA-AA diblock copolymer containing a short oligomeric PAA block.

Taking into account the above, the approximate molecular weight of the PAA block was calculated for the polymers whose GPC curves are shown in Fig. 4. The results are listed in Table 2 . The polymeric RAFT agent PBATC has dispersion $D$ not more than 1.16, which assumes blocks of this polymer having close molecular weight and containing the thio group in the middle of the chain. This assumption is also confirmed by the results of the model experiment presented above (Fig. 6). This makes it possible to consider the molecular weight of the PBA block, which is part of the BA-AA copolymer, as half the molecular weight of PBATC. For example, as follows from Table 2, upon the copolymerization of PBATC with $M_{\mathrm{n}}=30.4 \times 10^{3}$ and AA at a conversion of $6.9 \%$, the polymer with $M_{\mathrm{n}}=19.2 \times 10^{3}$ is formed. Accordingly, for the PBA block, $M_{\mathrm{n}}$ is $15.2 \times 10^{3}$, and for the PAA block, $M_{\mathrm{n}}$ is $4 \times 10^{3}$. The values of the molecular
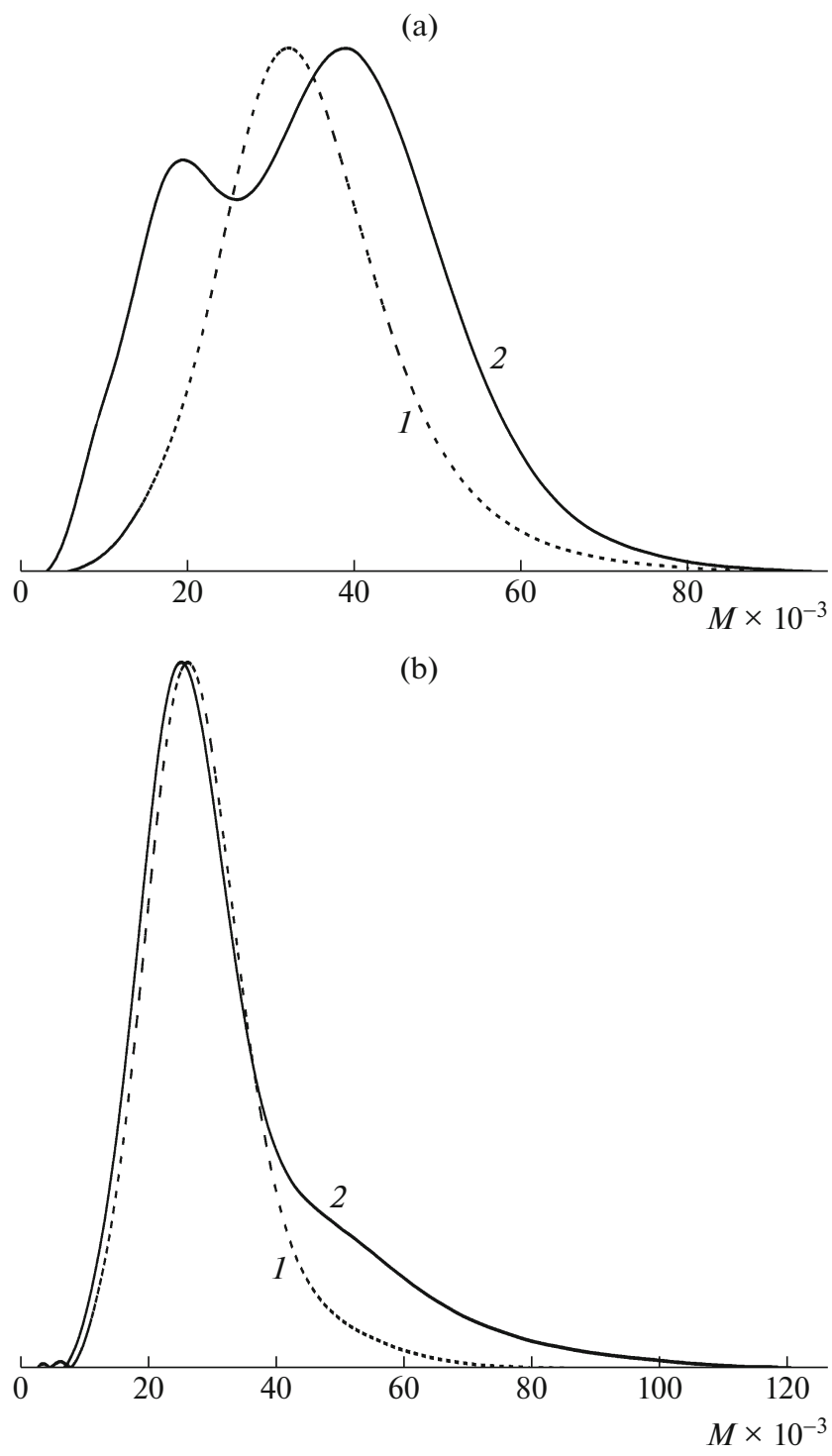

Fig. 6. GPC curves of (a) PBATC and (b) product of PBATC-mediated AA polymerization obtained (1) before and (2) after heating in benzene for $2.5 \mathrm{~h}$ in the presence of $0.1 \mathrm{~mol} / \mathrm{L}$ of AIBN. (a) [PBATC] $=1 \times 10^{-3} \mathrm{~mol} / \mathrm{L}$ and (b) $\left[\right.$ copolymer] $=1 \times 10^{-3}$ base-mol/L. $T=80^{\circ} \mathrm{C}$.

weight of the blocks calculated in this way are presented in Table 2. It can be seen that the PAA block has a low molecular weight characteristic of the oligomer $\left(3.9 \times 10^{3}\right)$, which is $2-4$ times lower than the molecular weight of the corresponding PBA blocks.

Thus, our data indicate that the asymmetric BAAA diblock copolymer is produced by the PBATCmediated polymerization of AA. The short length of the PAA block does not lead to a noticeable broadening of the MWD of the copolymer which is determined by a higher molecular weight narrowly dispersed PBA block. In the synthesis of the block copolymer with $M_{\mathrm{n}}=19.2 \times 10^{3}$ (Table 2), the value of $\boldsymbol{D}$ varies from 


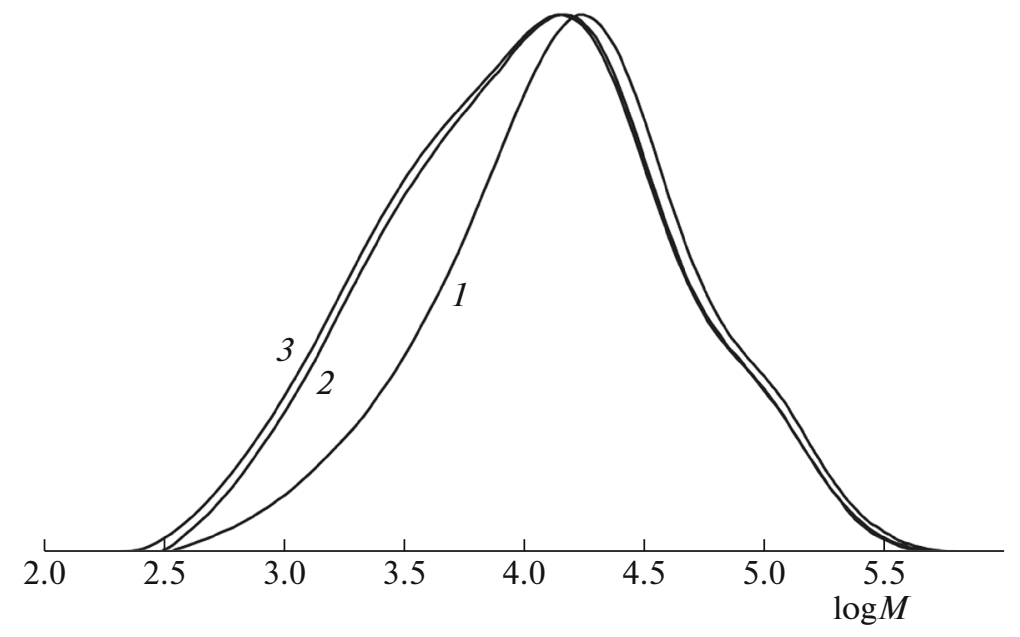

Fig. 7. GPC curves of $(1)$ the PBA homopolymer $\left(M_{\mathrm{n}}=10.5 \times 10^{3}, M_{\mathrm{w}}=37.8 \times 10^{3}\right)$ and the product of AA polymerization obtained in its presence after (2) 2 and (3) $3 \mathrm{~h}$ from the start of polymerization, the kinetic curve of which is shown in Fig. 4 (curve 3). (1) The initial concentration of components during BA polymerization, mol/L: $[\mathrm{BA}]=2.35$, $[\mathrm{IPA}]=8.73$, and $[\mathrm{AIBN}]=0.051 ; T=70^{\circ} \mathrm{C} ;(2,3)$ the initial concentration of components during AA polymerization mediated by PBA, mol/L: $[\mathrm{AA}]=0.36,\left[\mathrm{H}_{2} \mathrm{O}\right]=7.26,[\mathrm{IPA}]=11.07$, and $[\mathrm{APS}]=0.0024 ; 11.8 \mathrm{wt} \% \mathrm{PBA} ; T=70^{\circ} \mathrm{C}$.

1.18 for the initial PBATC to 1.12 for the block copolymer. However, as noted above, the absence of an increase in the molecular weight of AA with conversion, as well as of the thio group in the composition of the resulting polymer, indicates that PBATC does not participate in the polymerization of AA as an RAFT agent.

The conclusion is confirmed by comparative data on the effect of the low molecular weight RAFT agent DBTC and PBATC on the polymerization of AA. At initial concentrations of AA $(0.38 \mathrm{~mol} / \mathrm{L})$ and APS $(0.0009 \mathrm{~mol} / \mathrm{L})$ in the absence of additives, the rate of AA polymerization is $7.7 \%$ in $6 \mathrm{~h}$ and the molecular weight of the resulting PAA is $2.8 \times 10^{3}$. The addition of DBTC at the same concentrations of AA and APS causes a significant decrease in the molecular weight of PAA (380) and does not affect the polymerization rate (the yield of PAA is $4 \%$ in $6 \mathrm{~h}$ ). A similar effect of
DBTC is characteristic of the chain-transfer agent. At the same time, the introduction of PBATC instead of DBTC of the same concentration causes an increase in the rate of AA polymerization by a factor of 10 $(\sim 100 \%$ in $6 \mathrm{~h})$. In this case, the molecular weight of the PAA block (6370) calculated by the above procedure is on the same order of magnitude as the molecular weight of PAA obtained in the absence of additives (2800). These data suggest that the effect of PBATC on the polymerization of AA is atypical for the chaintransfer agent. Similar patterns are observed at other concentrations of the components.

To explain the mechanism of formation of the BAAA diblock copolymer, it is necessary to take into account the conditions of AA polymerization. The process takes place in the medium of the $\mathrm{H}$-donor IPA and in the presence of the radical initiator AIBN, that is, under conditions under which the replacement of

Table 2. Molecular weight characteristics and the proportion of high molecular weight and low molecular weight modes of the GPC curves as a function of conversion $P$ for the product of AA polymerization mediated by PBATC $\left(M_{\mathrm{n}}=30.4 \times 10^{3}\right.$, $\left.M_{\mathrm{w}}=35.7 \times 10^{3}, D=1.18\right)$

\begin{tabular}{c|c|c|c|c|c|c|c|c|c}
\hline \multirow{2}{*}{$P, \%$} & \multicolumn{4}{|c|}{ Basic mode } & PAA block & \multicolumn{3}{|c}{ Low molecular weight mode } \\
\cline { 2 - 9 } & $M_{\mathrm{w}} \times 10^{-2}$ & $M_{\mathrm{n}} \times 10^{-2}$ & $D$ & $S, \%$ & $M_{\mathrm{n}} \times 10^{-2}$ & $M_{\mathrm{w}} \times 10^{-2}$ & $M_{\mathrm{n}} \times 10^{-2}$ & $D$ & $S, \%$ \\
\hline 6.9 & 233.0 & 192.2 & 1.20 & 100 & 40.7 & - & - & - & - \\
14.2 & 228.6 & 191.3 & 1.20 & 100 & 39.8 & - & - & - & - \\
26.5 & 234.9 & 191.2 & 1.23 & 100 & 39.7 & - & - & - & - \\
60.0 & 220.4 & 190.0 & 1.16 & 99.5 & 38.5 & 27.0 & 24.7 & 1.10 & 0.5 \\
92.0 & 212.7 & 183.5 & 1.16 & 99.7 & 32.0 & 24.5 & 20.3 & 1.20 & 0.25 \\
98.0 & 210.7 & 187.5 & 1.12 & 97.4 & 36.0 & 30.7 & 25.1 & 1.22 & 2.6 \\
\hline
\end{tabular}

$S$ is the fraction of mode determined from the area of peaks. 
trithiocarbonyl groups in the molecules of the RAFT agent PBATC with hydrogen atoms of the IPA solvent is possible by the radical-induced reduction [21-23]. In particular, in accordance with this mechanism, terminal trithiocarbonate groups were removed from the RAFT agent $\left[\mathrm{NCCH}_{2}-\mathrm{PBA}-\mathrm{SC}(=\mathrm{S}) \mathrm{SC}_{12} \mathrm{H}_{25}\right]$ in a toluene-IPA mixture in the presence of the radical initiator dibenzoyl peroxide [21]. The NMR spectra of the isolated polymer reduction products confirmed the complete removal of the terminal thio groups in the polymer. It was suggested that the substitution gives rise to low molecular weight dodecyl trithiocarbonates and dithiobenzoates. However, the discoloration of polymer solutions observed in all cases indicates that these low molecular weight products are involved in further reactions involving the transformation of the $\mathrm{C}=\mathrm{S}$ group (owing to which trithiocarbonates are colored yellow).

In order to clarify the role of PBATC in the process of AA polymerization, a model experiment was carried out. A solution of DBTC $(0.0088 \mathrm{~mol} / \mathrm{L})$ with the initiator AIBN $(0.0177 \mathrm{~mol} / \mathrm{L})$ in IPA and also a solution of DBTC and AIBN taken at the same concentrations in benzene were heated at $70^{\circ} \mathrm{C}$ for $5 \mathrm{~h}$. The solutions were initially yellow. After heating, the solution in IPA became colorless, while the benzene solution retained its color. Both solutions after heating were studied by liquid chromatography. In the first solution, the initial concentration of the initial DBTC decreased to trace amounts $(0.8 \%)$, while in the second solution its content did not change. It was also shown that heating of the DBTC solution in IPA without the AIBN initiator does not lead to the disappearance of DBTC. Using these data and taking into account the results of [21], it can be assumed that radical-induced reduction takes place in the alcohol solution of DBTC and a similar process occurs during the PBATC-mediated radical polymerization of AA in the IPA-water mixed solvent. This is confirmed by the fact that, in all cases of AA polymerization mediated by PBATC, the reaction mixture lost its yellow color and became colorless, as in the experiment with the DBTC solution in IPA.

Let us consider a possible mechanism for the PBATC-mediated polymerization of AA monomer in IPA taking into account the reaction of the radicalinduced reduction of thiocarbonylthio compounds, the scheme of which is presented in [21, 22].

Initiation and propagation of PAA chains and chain transfer to the solvent

$$
\mathrm{AA}+\mathrm{I}^{\bullet} \rightarrow \mathrm{AA}^{\bullet} \stackrel{n \mathrm{AA}}{\longrightarrow} \mathrm{PAA}^{\bullet} \stackrel{\mathrm{HX}}{\longrightarrow} \mathrm{PAAH}+\mathrm{X}^{\bullet}
$$

Radical-induced reduction of PBATC

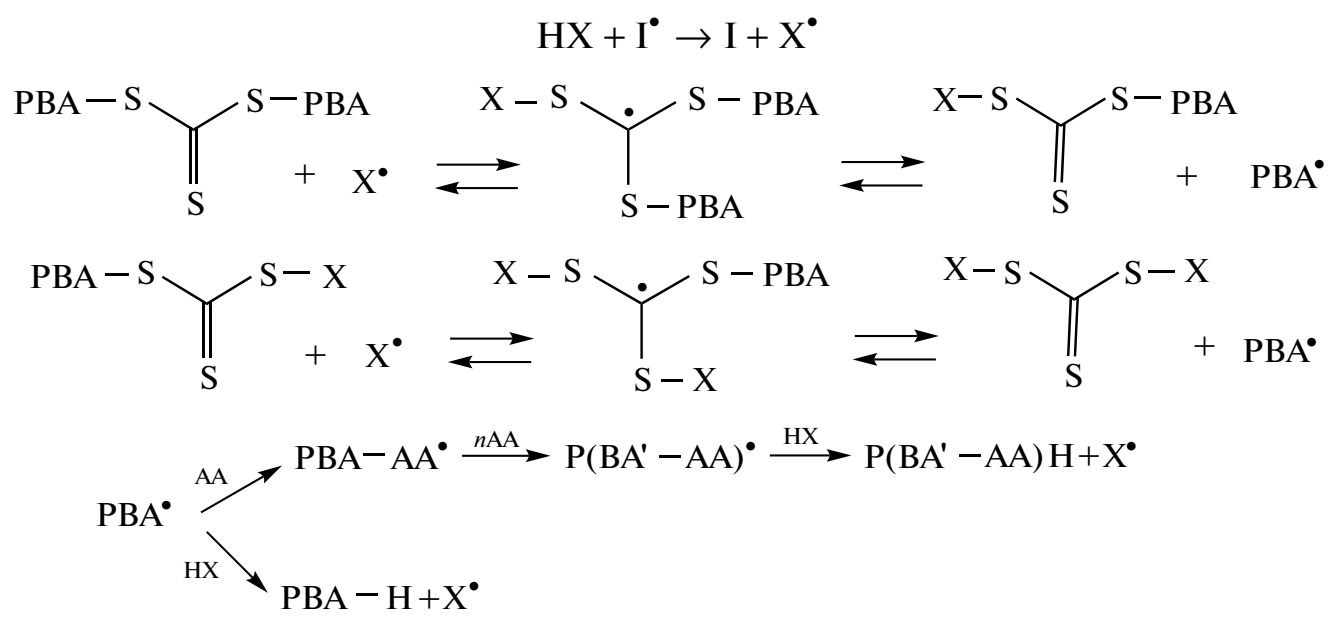

Chain termination

$$
\begin{aligned}
& \mathrm{PAA}^{\bullet}+\mathrm{PAA}^{\bullet} \rightarrow \mathrm{PAA}^{\circ} \\
& \mathrm{PBA}^{\bullet}+\mathrm{PBA}^{\bullet} \rightarrow \mathrm{PBA} \\
& \mathrm{PBA}^{\bullet}+\mathrm{PAA}^{\bullet} \rightarrow \mathrm{P}(\mathrm{BA}-\mathrm{AA}) \\
& \mathrm{P}\left(\mathrm{BA}^{\prime}-\mathrm{AA}\right)^{\bullet}+\mathrm{P}\left(\mathrm{BA}^{\prime}-\mathrm{AA}\right)^{\bullet} \rightarrow \mathrm{P}(\mathrm{BA}-\mathrm{AA}) \\
& \mathrm{P}\left(\mathrm{BA}^{\prime}-\mathrm{AA}\right)^{\bullet}+\mathrm{PAA}^{\bullet} \rightarrow \mathrm{P}(\mathrm{BA}-\mathrm{AA})
\end{aligned}
$$

$$
\mathrm{P}\left(\mathrm{BA}^{\prime}-\mathrm{AA}\right)^{\bullet}+\mathrm{PBA}^{\bullet} \rightarrow \mathrm{P}(\mathrm{BA}-\mathrm{AA}-\mathrm{BA})
$$

Here $\left.\mathrm{X}^{\bullet}=\left(\mathrm{CH}_{3}\right)_{2}-\mathrm{COH}\right)^{\bullet}$ and $\mathrm{HX}=$ IPA.

Reaction (I) is the formation of acrylic acid homopolymer. It includes the stage of initiation, chain propagation, and chain termination on the $\mathrm{H}$-donor molecule (IPA). The second reaction refers to interaction of the initiator radical with the $\mathrm{H}$-donor molecule (IPA) which generates radicals $\left(\mathrm{X}^{\bullet}\right)$ that can replace PBA macroradicals in the PBATC molecule. As a result, two PBA macroradicals and a low molecular 


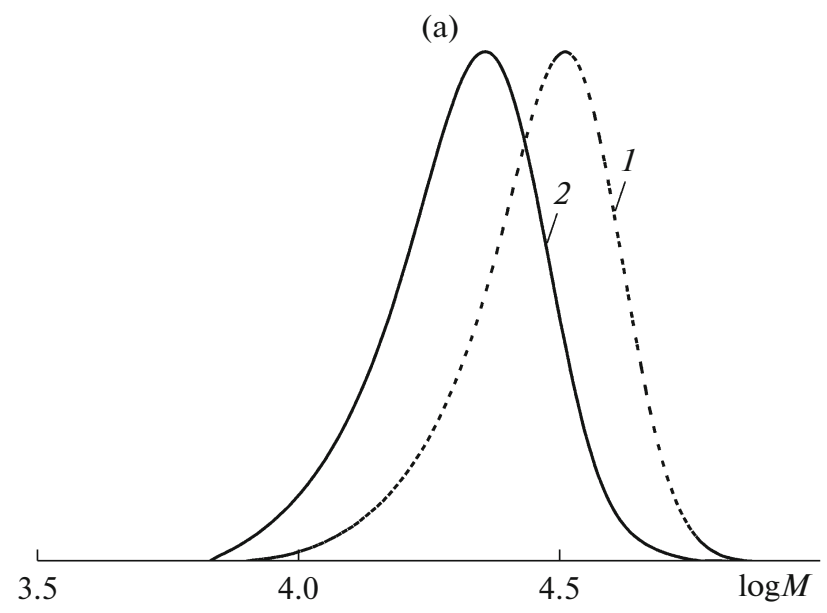

(b)

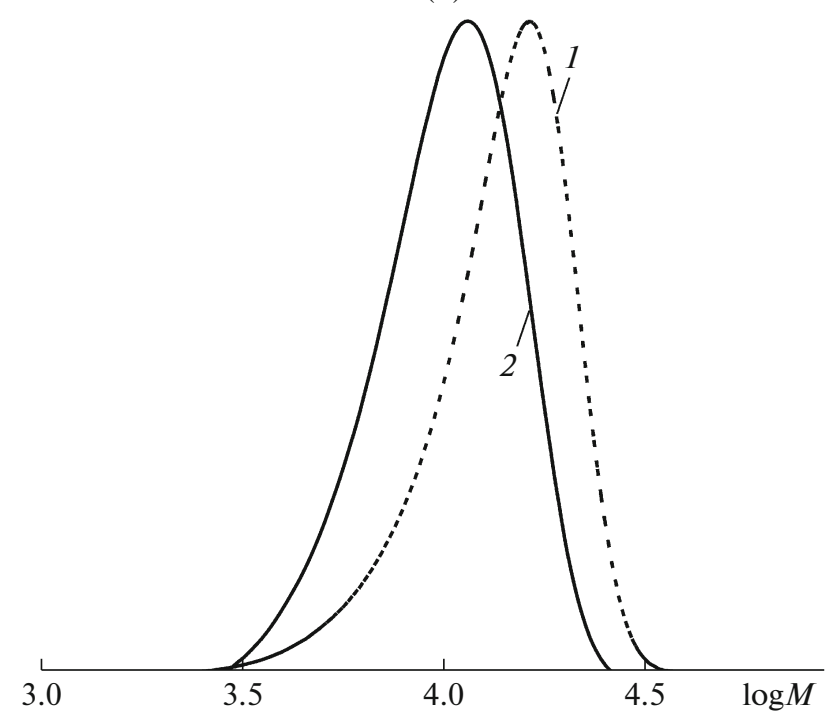

Fig. 8. GPC curves of (1) the initial PBATC and (2) the copolymer BA-AA. $M_{\mathrm{n}}=$ (1) 28280 and (2) (a) 20510 and (b) 13110

weight trithiocarbonate are formed from one PBATC molecule (reactions (III), (IV)). The mechanism of its disappearance (as a result of which the discoloration of the reaction mixture occurs) remains unclear [21, 22].

PBA macroradicals can be involved in competing reactions: copolymerization with acrylic acid to form the BA-AA diblock copolymer; chain transfer to solvent HX (IPA); and bimolecular chain termination.

As follows from the above scheme, owing to bimolecular termination reactions (VII), (IX), and (XI), the (co)polymer with a molecular weight 2 times higher than that of the initial PBATC should be formed. At the same time, according to termination reactions (VI), (VIII), and (X), as well as reactions of chain transfer to IPA (reactions (I) and (V)), the molecular weight of the (co)polymer should be comparable to the molecular weight of PBATC. This should lead to a bimodal MWD of the product of AA polymerization mediated by PBATC, as was observed in [21] upon the removal of trithiocarbonate groups from the RAFT agent $\left[\mathrm{NCCH}_{2}-\mathrm{PBA}-\right.$ $\mathrm{SC}(=\mathrm{S}) \mathrm{SC}_{12} \mathrm{H}_{25}$ ] in toluene. However, as was shown in [21], the bimolecular termination of macroradicals cannot compete with the radical-induced reduction upon addition of a stronger chain-transfer agent, IPA, to toluene. The unimodal MWD of the obtained PBA was used as proof. In our case, the MWD of the copolymers is also unimodal (Figs. 4, 8). In this case, the main competing reactions are the interaction of the PBA macroradical with acrylic acid accompanied by formation of the BA-AA diblock copolymer (reaction (Va)) and chain transfer to the HX solvent (IPA) (reactions $(\mathrm{I})$ and $(\mathrm{Vb})$ ). It follows from the scheme that the PAA homopolymer is formed via reaction (I). As shown above, the main mode on the GPC curves of the AA polymerization product obtained in the presence of PBATC refers to the BA-AA block copolymer (Fig. 4). The low molecular weight mode seen in Fig. 4 apparently belongs to the PAA homopolymer, but it does not manifest itself in all experiments (Fig. 8). The possibility of its elimination is most likely associated with selection of the optimal ratio between the initiator and PBATC, which will be studied in the future.

Let us consider the probability of the main competing reactions $(\mathrm{Va})$ and $(\mathrm{Vb})$. In the copolymerization of PBA with AA, the reaction rate is described by the equation $v_{12}=k_{12} \times\left[\mathrm{PBA}^{*}\right] \times[\mathrm{AA}]$. For this monomer pair, it follows from their reactivity ratios [24] that the PBA macroradical is equally active with respect to both its monomer and AA. This makes it possible to use in calculations instead of $k_{12}$ the rate constant of the reaction of BA chain propagation in its homopolymerization $\left(k_{11}\right)$, which is $2.8 \times 10^{4} \mathrm{~L} /(\mathrm{mol} \mathrm{s})$ [24]. The minimum used concentration of AA is $0.38 \mathrm{~mol} / \mathrm{L}$; the stationary concentration of PBA is $\sim 10^{-8} \mathrm{~mol} / \mathrm{L}$ [25]. As a result of calculations, we arrive at $v_{12} \sim 1.1 \times 10^{-4} \mathrm{~mol} /(\mathrm{L} \mathrm{s})$.

The activating effect of water on the polymerization of AA is known [26]. Therefore, to estimate the rate constant of chain propagation from the published data, conditions were chosen that were as close as possible to the conditions of polymerization in our study. Using the data from [27] devoted to the $\mathrm{K}_{2} \mathrm{~S}_{2} \mathrm{O}_{8}$-initiated polymerization of $\mathrm{AA}$ at $50^{\circ} \mathrm{C}$ in a methanolwater mixture of different composition, the rate constant of chain propagation $k_{\mathrm{p}}$ can be estimated as $4 \times$ $10^{4} \mathrm{~L} /(\mathrm{mol} \mathrm{s})$. According to [28], $k_{\mathrm{p}}$ for BA at $50^{\circ} \mathrm{C}$ is on the same order of magnitude $\left(2.8 \times 10^{4} \mathrm{~L} /(\mathrm{mol} \mathrm{s})\right)$. It is also known [24] that the chain-transfer constant $C_{\text {tr }}$ for (meth)acrylates to lower alcohols is not more than $10^{-3}$. Then the rate of interaction of PBA and PAA macroradicals with IPA molecules should not exceed $10^{-5} \mathrm{~mol} /(\mathrm{L} \mathrm{s})$. 
Thus, the rate of interaction of PBA macroradicals with the AA monomer is significantly higher than the rate of chain transfer of the PBA macroradicals to the IPA solvent (by about an order of magnitude). Accordingly, this leads to the fact that the main product of the AA polymerization mediated by PBATC in the IPA-water medium is the BA-AA diblock copolymer.

The proposed method of the homophase copolymerization of AA with PBATC in the mixed solvent IPA $-\mathrm{H}_{2} \mathrm{O}$ was used for the synthesis of transparent solutions containing from 10 to $35 \%$ of the block copolymer BA-AA. The resulting concentrated solutions were diluted with a mixed solvent of the required composition to a concentration of $2 \%$ and investigated by dynamic light scattering. It was found that the solutions contain nanoparticles. Figure 9 shows the dependence of the particle size in $2 \%$ colloidal solutions of the BA-AA diblock copolymer on the water content in the used binary solvent. The copolymer with $M_{\mathrm{n}}=$ $17.3 \times 10^{3}$ was obtained by the polymerization of AA mediated by PBATC $\left(M_{\mathrm{n}}=21.2 \times 10^{3}, \nexists=1.12\right)$. The initial concentration of components, mol/L: $[\mathrm{AA}]=$ $3.3,[$ PBATC $]=0.0064,\left[\mathrm{H}_{2} \mathrm{O}\right]=4.59,[\mathrm{IPA}]=9$, and $[\mathrm{APS}]=0.005$.

As follows from Fig. 9, with an increase in the content of water (precipitant for PBA) in the mixed solvent from 13.4 to $50 \%$, the particle diameter increases from 11.7 to $42.6 \mathrm{~nm}$. This indicates that the size of particles in the colloidal solution of the BA-AA block copolymer depends on the solubility of the PBA coreforming blocks. Figure 9 also shows that the colloidal solutions contain one type of nanoparticles with a narrow size distribution. To make sure that the detected particles were not formed by PAA macromolecules, PAA solutions with $M=2.8 \times 10^{3}$ in the mixed solvent IPA-water with different water content were investigated. It was found that the PAA solutions at a water concentration of less than $35 \%$ contained particles whose size did not exceed $1-5 \mathrm{~nm}$, that is, the size of individual macromolecules. At a water content in the mixed solvent of $35 \%$ or higher, particles $300-400 \mathrm{~nm}$ in size were observed. In the colloidal solutions of the BA-AA block copolymer, particles of this size were not detected.

\section{CONCLUSIONS}

Thus, the use of the mixed solvent (2-propanolwater) in the synthesis of the BA-AA block copolymer from PBATC and AA, the thermodynamic quality of which with respect to one of the copolymer blocks deteriorates during synthesis from a good solvent to a precipitant under normal conditions (near the solubility limit), makes it possible to obtain colloidal solutions with a block copolymer particle diameter of 10$100 \mathrm{~nm}$. The colloidal solution of the BA-AA block copolymer obtained by the heterophase method in a

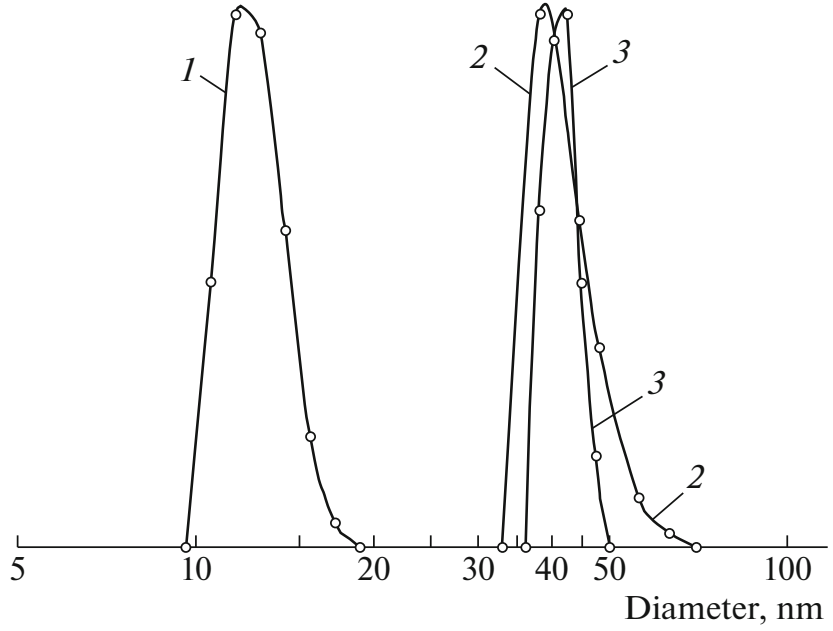

Fig. 9. Particle diameter distribution in the colloidal solution of the BA-AA copolymer in the mixed IPA-water solvent (precipitant). Water content: (1) 13.4, (2) 33, and (3) $50 \%$; the concentration of the BA-AA copolymer is 2 wt $\% ; M_{\mathrm{n}}=17.3 \times 10^{3} ; T=70^{\circ} \mathrm{C}$.

similar medium using a PAA-based RAFT agent contains nanoparticles with a diameter of more than $200 \mathrm{~nm}$ [29]. Unlike the heterophase method, in the proposed method of obtaining the colloidal solution, the reaction medium under the conditions of synthesis is lyophilic both with respect to the polymeric RAFT agent and with respect to the resulting second block of the block copolymer. This eliminates the need to use a stabilizer during synthesis and potentially allows by preliminary selection of the solvent for the blocks of the synthesized copolymer under normal conditions to target the micelle structure, determining in advance which of the blocks will form the core of particles and which will form their shell.

The mechanism governing formation of the narrowly dispersed diblock copolymer of butyl acrylate with acrylic acid is proposed, according to which the polymeric RAFT agent is involved in the reaction of radical-induced reduction with the solvent 2-propanol and generates poly(butyl acrylate) macroradicals which interact with acrylic acid to afford the narrowly dispersed diblock copolymer.

\section{OPEN ACCESS}

This article is distributed under the terms of the Creative Commons Attribution 4. International license (http://creativecommons.org/licenses/by/4.0/), which permits unrestricted use, distribution, and reproduction in any medium, provided you give appropriate credit to the original author(s) and the source, provide a link to the Creative Commons license, and indicate if changes were made. 


\section{REFERENCES}

1. S. L. Canning, C. N. Smith, and S. P. Armes, Macromolecules 49, 1985 (2016).

2. C. L. McCormick and A. B. Lowe, Acc. Chem. Res. 37, 312 (2004).

3. R. G. Chaudhuri and S. Paria, Chem. Rev. 112, 2373 (2012).

4. N. J. Warren and S. P. Armes, J. Am. Chem. Soc. 136, 10174 (2014).

5. W. D. He, H. L. Sun, W. M. Wan, and C. Y. Pan, Macromolecules 48, 3358 (2015).

6. Y. Pei, L. Thurairajah, O. R. Sugita, and A. B. Lowe, Macromolecules 48, 236 (2015).

7. Y. Pei, J. M. Noy, P. J. Roth, and A. B. Lowe, J. Polym. Sci., Part A: Polym. Chem. 53, 2326 (2015).

8. M. J. Derry, L. A. Fielding, and S. P. Armes, Polym. Chem. 6, 3054 (2015).

9. H. Zhou, C. Liu, C. Gao, Y. Qu, K. Shi, and W. Zhang, J. Polym. Sci., Part A: Polym. Chem. 54, 1517 (2016).

10. C. Gao, H. Zhou, Y. Qu, W. Wang, H. Khan, and W. Zhang, Macromolecules 49, 3789 (2016).

11. V. N. Kuleznev, Polymer Blends (Khimiya, Moscow, 1980) [in Russian].

12. N. Yu. Shushunova, B. B. Troitskii, S. A. Chesnokov, T. A. Kovylina, M. V. Arsenev, A. N. Konev, M. Yu. Zakharina, and I. L. Fedyushkin, Russ. J. Appl. Chem. 88, 1667 (2015).

13. W. M. Lee Albert, W. H. Chan, and H. C. Wong, Synth. Commun. 18, 1531 (1988).

14. E. V. Vasilyeva, N. A. Kopylova, S. D. Zaitsev, and Yu. D. Semchikov, Polym. Sci., Ser. B 53, 491 (2011).

15. A. A. Tager, Physics and Chemistry of Polymers (Khimiya, Moscow, 1978) [in Russian].
16. V. F. Alekseev, Zh. Russ. Khim. O-va 9, 208 (1877).

17. W. H. Stockmayer and M. Fixman, J. Polym. Sci., Part C: Polym. Symp., No. 1, 137 (1963).

18. E. V. Chernikova, Doctoral Dissertation in Chemistry (MGU, Moscow, 2010).

19. F. D. Kuchta, A. M. Herk, and A. L. German, Macromolecules 33, 3641 (2000).

20. N. M. Ahmad, F. Heatley, and P. A. Lovell, Macromolecules 31, 2822 (1998).

21. J. K. Chong, G. Moad, E. Rizzardo, and S. H. Thang, Macromolecules 40, 4446 (2007).

22. G. Moad, E. Rizzardo, and S. H. Thang, Polym. Int. 60, 9 (2011).

23. S. Perrier, Macromolecules 50, 7433 (2017).

24. Yu. S. Lipatov, A. E. Nesterov, T. M. Gritsenko, and R. A. Veselovskii, Handbook on Polymer Chemistry (Naukova dumka, Kiev, 1971) [in Russian].

25. G. Odian, Principles of Polymerization (McGraw-Hill Book Company, New York, 1970).

26. V. A. Kabanov, V. P. Zubov, and Yu. D. Semchikov, Complex-Radical Polymerization (Khimiya, Moscow, 1987) [in Russian].

27. I. M. Papisov, V. A. Kabanov, E. Osada, M. Leskano Brito, Zh. Raimont, and A. N. Gvozdetskii, Vysokomol. Soedin., Ser. A 14, 2462 (1972).

28. Fundamentals of Controlled/Living Radical Polymerization, Ed. by N. V. Tsarevsky and B. S. Sumerlin (RSC, Dallas, 2013).

29. T. V. Chernikova, A. V. Plutalova, K. O. Mineeva, I. R. Nasimova, E. Yu. Kozhunova, A. V. Bolshakova, A. V. Tolkachev, N. S. Serkhacheva, S. D. Zaitsev, N. I. Prokopov, and A. B. Zezin, Polym. Sci., Ser. B 57, 547 (2015). 